\title{
FUNDAMENTAL FORCES AND UNIFIED FIELD THEORY
}

\author{
Dhananjay Kumar Tiwary \\ Department of Mathematics Education
}

\begin{abstract}
This paper deals with fundamental particles, their classifications on the basis of Bose - Einstein Statistics, Fermi- Dirac Statistics and Quark Theory along with four types of existing fundamental forces. This also reflects on unified field theory regarding unification of the fundamental forces.
\end{abstract}

\section{Keywords}

Bosons, fermions, quarks, Higgs' particles, standard model

\section{Introduction}

During early 1930s, atomic theory included only protons, neutrons and electrons as the fundamental particles. Any matter is built up of fundamental particles. At present, about more than 34 fundamental particles are known. Most of the particles can be described as constituting either matter or anti-matter on the basic of relativistic quantum mechanics.

Albert Einstein pointed out that light (radiation) has also nature of particle (Photo Electric Effect). This particle of light is known as 'light quanta' or 'photon'. Such a particle has zero rest mass and speed of light. It has $\mathrm{a} \pm 1$ spin and it can be produced or absorbed by an oscillating electrical dipole. Similarly, it follows that a system of two masses such as the earth and the moon, rotating along a common centre axis would emit gravitational quanta or graviton $(G)$. This particle has also zero mark and a speed of light (Fadnis, 1986).

Murray Gellmann, known as 'Mendeleev of physics', has classified the particle-world into two families: Leptons and Hadrons. The Leptons are elementary in nature where the hadrons are nothing but the different combinations of special kind of elementary particles called 'Quark'. Gell- Mann, named fundamental particles as 'Quarks' and their counterpart as 'Anti-quarks'. George Zweing of California Institute of Technology, USA, independently arrived at a common concept and named them as 'ACES' and 'ANTI - ACES'.

According to Quark theory, the sub-nuclear particles (Hadrons) are composed of Quark and anti - quark. Quarks are considered to be point-like, possibly dimensionless particles. The present theory accepts six quarks - (i) up 
(u) ii) down (d) iii) strange (s) iv) charmed (c) v) truth (t) vi) beauty (b) and six anti - quarks. Thus, Quark theory provides twelve fundamental particles. A proton is made of a combination of two u-quarks and one d-quark. Similarly, the neutron consists of two d-quarks and one u-quark. The quarks bear a special property that it can't exist freely in nature. If we try to separate the quarks present in the proton as their separation increases the attraction among them and they will become stronger. On the other hand, if they are very close to each other, they exhibit a free nature. This property of quark is called 'Asymptotic Freedom'. Each of the quarks is electrically charged and also bears a special kind of charge, called 'Colour Charge'. There are three kinds of colour charges - red, blue and green (Bhattacharyya, 2009).

Apart from the above classification, the fundamental particles can also be categorized into two classes on the basis of magnitude of their spin- Bosons and Fermions.

Now, the question arises - what is spin? The intrinsic spin of a particle is expressed by a quantum number known as 'spin quantum number', denoted by $\mathrm{S}$. In terms of spin quantum number; the snin anoular

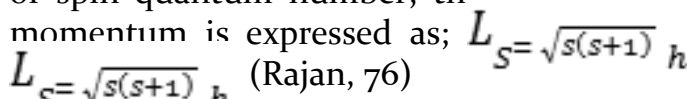

The spin quantum number $\mathrm{S}$ takes half integral values for 'Fermions'. These particles follow Fermi - Dirac statistics, named after physicist Enrico Fermi. These fermions are described by anti - symmetric wave - function. But the particles having integral spin follows a statistics called Bose - Einstein statistic, proposed jointly by the Indian scientist $\mathrm{S}$. N. Bose and Albert Einstein, is called 'Boson'. Bosons are described by symmetric wave functions. The Higg's particle also belongs to the 'Boson' category as it has the spin zero (considered as integral spin).

Nowadays, Large Hadrons Colliders (LHC) is a burning topic in newspapers and electronic media. One of the main objectives of LHC experiment is to catch the Higg's particle, of course, if it exists. If the Higg's particle is found in the LHC experiment, it will prove the fusibility of the 'Standard Model Theory', not the existence of God. This standard model was given by three scientists Glashow, Salam, and Weinberg in 1979. These scientists also won the Noble Prize in Physics for the unification of electromagnetic and weak interactions of the sub - atomic particles (Bhattacharyya, 2009).

\section{Forces between fundamental particles}

Fundamentally, four types of forces exist in the universe. They are: i) Strong force Electromagnetic force iii) Weak force Gravitational force.

Strong Force: it is the strongest known force responsible for bounding proton to neutron and quarks to quarks. It is $\sim 100$ times greater than the electrical force and has a range $10^{-13} 10^{-13} \mathrm{~cm}$ which is approximately equal to the diameter of 'Hadron'.

Electromagnetic force: it is a stronger force existing between two charged objects. It has an infinite range and is effective on the macro as well as subatomic particles.

Weak Force: The weak force is responsible for the decay of neutrons into protons and electrons, called $\beta$ - decay. It is very much weaker $\left(\sim 10^{-11} 10^{-11}\right.$ times $)$ than the electrical force and has a very short range $\sim 10^{-15} 10^{-15} \mathrm{~cm}$. It can act on quarks and leptons and brings about particles transmutation in the form of particles decay. To explain the nature of the electron spectrum in $\beta$ - decay, Pauli theoretically deducted a new kind of mass-less, charge-less, known as neutrons. It is not responsible for any kind of electromagnetic interaction. But it plays a significant role in the weak interaction. Actually, the weak interaction is originated by the exchange of massive boson particles between two special kinds of particles. Such massive vector bosons are of three types; electrically positive $\left(w^{+} w^{+}\right)$, electrically negative $\left(w^{-} w^{-}\right)$, and neutral $(\mathrm{z})$. 
Gravitational force: It is the weakest of all the forces and has infinite range. It is significant only between the bodies having large masses and therefore, is of little significance at the subatomic level.

These four fundamental forces are the 'Interactions' among different particles in the quantum level. In our daily life, the forces experienced are either electromagnetic or gravitational in nature. The other two kinds of forces exist in the nuclei of atom.

\section{Unified field theory}

In eighteenth century, some physicists and mathematicians attempted to develop a single theoretical framework that can account for the fundamental forces of nature, which came to be known as a unified field theory. The early attempts began with the Riemannian geometry of general relativity. The first attempts to propound a unified theory were by G. Mie in 1912 and Ernst Reichenbacher in 1916. However, these theories were unsatisfactory. Between 1918 to 1923 , there were four distinct approaches to field theory: the gauge theory of Weyl, Kaluz's five dimensional theory, Lancelot Law Whyte's theory based on the unitary principle and Eddingtan's development affine geometry.

The relation $\mathrm{E}=\mathrm{mc} 2 m c^{2}$ cotributes between mass and energy was first established by Albert Einstein. It contributes to the stress tensor and thus to the curvature of space-time, which is the general-relativistic representation of the gravitational field. Einstein tried to form a generalized theory of gravitation that would unify the gravitational and electromagnetic forces (and perhaps others) guided by a belief in a single origin for the entire set of physical laws. He sought to relate the universal properties of matter and energy in the single equation or formulae, known as "unified field theory". However, this turned out to be a fruitless quest due to uncertainty principle of Quantum theory which states that the movement of single electron simply could not be predicted because its position and (speed) at any instant could not be measured with equal precision (Fadnis, 1986).

Max Born, a distinguished German Quantum theorist, said at the time; "Many of us regard this as a tragedy, both for him as he gropes his way in loneliness, and for us, who miss our leader pronouncing his work in later life as largely wasted effort, will have to await the judgment of later generations". Einstein himself also stated, "I cannot prove that I am right in this, but that is my religion". (Barnett, 1957)

Abbe Georges Lemaitre, in his 'Big Bang Theory', cites that universe had been created by the explosion of a "primeval atom" and was still expanding. The fundamental particles and the forces 'froze out' as products on the cooling of universe. Conversely, if energy is gradually decreased, the particles can be transformed into force and then unification of all these force can be visualized. Gleefully, Einstein jumped to his feet applauding, "this is the most beautiful and satisfactory explanation of creation to which I have ever listened", he said. (Barnett, 1957).

The rejection of his ideas by statesman and politicians did not break him because his prime obsession still remained with physics, "I cannot tear myself away from my work" he wrote at that time, "it has me inexorable in its clutches. "In proof of this came his new version of the "unified field theory" in 1950s, a most meticulous mathematical essay that was immediately but politely criticized by most physicists as untenable.

On April 18, 1955, Einstein died leaving his last incomplete statement "what I seek to accomplish is simply to serve with my feeble capacity, truth and justice at the risk of pleasing no one". He also said, "politics are for the moment, an equation is for eternity" (Barnett, 1957).

Einstein is no more but Max Born forecasting sounds true. Nowadays it is accepted that all the particles and forces have a common starting point in that all the fundamental particles and forces acting between them are 
originally the same or unified. Recent work by Komech (Komech,2005) puts Einstein's earlier work in a more favorable light. This book realizes Einstein's Unified Field Theory program and shows that almost all the empirical result known until the 1980 os were derivable from it.

In 1965, Hoyle proposed his "Steady state theory". According to him, a matter is continually created out of energy and continually destroyed at a constant rate, thus, maintaining a balance in the expanding universe. However, now it is believed that the universe is in a state of constant flux, i.e. expanding outward and contracting inward in accordance with the widely held Big Bang theory.

In 1967, Steven Weinberg and Abdus Salam working independently hypothetically unified the weak and electrical forces into a single entity - "The Electroweak force". It can now be assumed that the addition of energy would first separate the hadrons from each other followed by separation of quarks from one another. At some point, the quarks and leptons would become interchangeable and finally the particles and forces become indistinguishable. The model given by Glashow, Salam and Weinberg is commonly known as the 'Standard Model'. According to this model, all the leptons and quarks are also mass-less and below the symmetry breaking scale they have their respective masses. To establish any theory, experimental verification is necessary. The standard model predicts the existence of new particles like $w^{+} w^{+}$, $w^{-} w^{-}, \mathrm{z}$ and Higgs bosons (after the name of scientist Peter Higgs). Although all the other particles have been found, the existence of Higgs Boson is yet to be experimentally confirmed.

On theoretical consideration, almost every event can be explained if enough energy is provided; consequently, one can view the fundamental particles and forces of nature as a unified picture with the same origin. All the recent researches are being directed towards giving experimental basis for theoretical proposals. Current researches on unified field theories focus on the problem of creating quantum gravity and unifying such a theory with the other fundamental theories i.e. quantum theories. With four fundamental forces now identified, gravity remains the one force whose unification proves problematic.

\section{References}

Bhattacharya, I. (2009). The LHC experiment - Science versus Rumors -Breakthrough. A Journal on science and society, 13 (4), pp. 4 - 10.

Colder, N. (1981) The key to this Universe. New York : Penguin Books.

Fadnis, A. G. (1986). Fundamental particles. Science Reporter, Vol. 23 No. 02, 89 - 92.

Halzen, F. \& Martin, A. D. (1984). Quarks and Leptons: An introductory course to modern particles physics. New York : Wiley.

Kinger, N. (2010). The life and works of Albert Einstein. New Delhi: Sahni Publications.

Komech, A. I. (2005). Lectures on Quantum Mechanics (nonlinear PDE point of view). Retrieved on 12 April,2011 from http://arxiv. org/PS_cache/mathph/pdf10505/0505059v4.pdf

Lincoln B. (1957). The Universe and Dr. Einstein. New York: Bantum c/o William Marrow and co. Inc.

Rajan, J. B. (1976) Atomic Physics, New Delhi : S. Chand and Company.

\section{Website}

http://www.gradian.co.uk/science/20o8/ jan/3o/higgs.boson.cern.

\section{The Author}

Dhananjay Kumar Tiwari is an Associate Professor in Maths Education in Thakur Ram Multiple Campus, Birgunj. He has been teaching courses in Mathematics for the last twenty three years. His subjects of interest are Real Analysis and Topology. He is a life member of Council for Mathematics Education. 